\title{
МАТЕМАТИЧЕСКОЕ МОДЕЛИРОВАНИЕ РЕЖИМОВ РАБОТЫ КОЛЕБАТЕЛЬНОЙ ШАРОВОЙ МЕЛЬНИЦЫ
}

\author{
Данекер Валерий Аркадьевич, \\ vad@tpu.ru \\ Национальный исследовательский Томский политехнический университет, \\ Россия, 634050, г. Томск, пр. Ленина, 30.
}

\begin{abstract}
Актуальность настоящего исследования обусловлена необходимостью создания высокоэффективного ресурсосберегающего оборудования, применяемого при дроблении и измельчении рудного материала в горнодобывающей промышленности. Одним из путей создания такого оборудования является применение шаровых мельниц колебательного типа, в конструкции которых используется тяговый электромагнит переменного тока. Подключение тягового электромагнита к источнику напряжения переменной частоты позволяет регулировать режимы работы шаровой мельницы в широких пределах и обеспечивать её работу в квазирезонансных режимах. Математическое моделирование режимов работы шаровой мельницы колебательного типа позволяет определить требования к параметрам колебательной системы при варьировании частоты и величины питающего напряжения и разработать практические рекомендации, которые следует учитывать при проектировании промышленных образцов. Кроме этого, функционирование шаровой мельницы колебательного типа в квазирезонансных режимах сопровождается существенным сокращением энергозатрат при увеличении производительности.

Цель исследования: разработка математической модели шаровой мельницы колебательного типа при питании тягового электромагнита от источника напряжения переменной частоты; на основе разработанной математической модели провести расчёты режимов работы шаровой мельницы колебательного типа и сформулировать практические рекомендации для использования при проектировании шаровой мельницы.

Методы. Математическое моделирование режимов работы шаровой мельницы проводилось на основе численного решения системы дифференциальных уравнений движения элементов колебательной системы. Параметры колебательной системы определялись расчётным путём с учётом реальных технических характеристик выпускаемого аналогичного оборудования.

Результаты. Математическая модель шаровой мельницы колебательного типа позволяет определить диапазоны тяговых усилий и частот напряжения питания тягового электромагнита, соответствующие квазирезонансному режиму работы при изменении параметров колебательной системы. На основе установленного диапазона квазирезонансных частот и масс элементов колебательной системы могут быть определены параметры тягового электромагнита, конструкция и параметры пружинного подвеса шаровой мельницы.
\end{abstract}

\section{Ключевые слова:}

Руда, дробление, измельчение, шаровая мельница, вибрационная мельница, колебательная система, тяговый электромагнит, резонанс.

\section{Введение}

Дробление и измельчение являются одними из основных процессов при переработке руды в горнодобывающей и горно-перерабатывающей отраслях. На долю этих процессов, по некоторым данным, приходится до 70 \% энергозатрат. Вследствие этого разработка нового и совершенствование существующего оборудования, применение новых подходов к данным процессам, решающих задачи снижения энергозатрат, материалоёмкости, повышения производительности являются своевременными и актуальными [1-5].

Значительную часть оборудования одного из этапов обработки руды составляют шаровые мельницы (ШМ). Разрушение конгломератов рудного материала (дробление, измельчение) в ШМ происходит за счёт их взаимодействия с мелющими телами (шарами), друг с другом и с элементами конструкций помольной камеры собственно ШМ. Затраты электроэнергии приводного двигателя ШМ составляют от $45,0 \mathrm{kB}$.ч и выше на тонну готового продукта, что является достаточно энергозатратным процессом, требующим совершенствования [6-9].

Анализ технических характеристик имеющегося в настоящее время парка ШМ показывает, что соотношение объёмов загружаемого материала с мелющими телами не превышает 50 \% от общего объёма барабана, что свидетельствует о высокой материалоёмкости эксплуатируемых в настоящее время конструкций ШМ.

Одним из распространённых типов ШМ являются мельницы вибрационного типа. Исследование эксплуатационных характеристик ШМ вибрационного типа (ШМВТ) с циклической загрузкой и выгрузкой показывает, что их применение позволяет снизить затраты электроэнергии при увеличении производительности [10].

Вибрация ШМВТ возникает за счёт вращения дебаланса, создающего круговые колебания помольной камеры с размещёнными в ней мелющими телами и рудным материалом. Амплитуды колебаний корпуса такой ШМ составляют несколько миллиметров. Для снижения вибрационной нагрузки на окружающее пространство корпус ШMВТ, как правило, размещают на пружинном подвесе [11, 12].

Центробежная сила круговых колебаний вызывает существенную паразитную механическую нагрузку на электродвигатель, корпусные элементы конструкции ШМВТ и на пружины подвеса. Вследствие этого ресурс и надёжность ШМВТ не 
высоки, что особенно ограничивает перспективы производства крупногабаритных ШМВТ. Кроме этого, использование дебаланса для создания вибраций не позволяет реализовать раздельное регулирование значения вибрационной силы и частоты круговых колебаний. $\mathrm{K}$ тому же отсутствуют сведения о практическом использовании каких-либо принципов регулирования режимов работ ШМВТ.

Создание колебаний помольной камеры ШМ строго в одной плоскости позволяет избежать вышеперечисленных недостатков ШМВТ. Реализация колебаний строго по одной пространственной координате возможна при использовании тяговых электромагнитов (ТЭМ) переменного тока соленоидного или клапанного типов. Изменяя величину напряжения, подаваемого на обмотку ТЭМ, можно регулировать в широких пределах величину вынуждающей силы колебательной системы, влияющей на производительность и дисперсность выходного продукта ШМ такого принципа работы. Вторым самостоятельным каналом регулирования режимов работы электромагнитной ШМ колебательного типа (ЭШМКТ), не связанным с предыдущим вариантом, является возможность регулирования частоты возмущающей силы, которая пропорциональна частоте питающего напряжения. Несомненным достоинством ЭШМКТ является и то обстоятельство, что в отличие от ШМВТ с дебалансом в них появляется возможность реализовывать режимы работы, близкие к состоянию резонанса колебательной системы при отсутствии паразитных механических нагрузок. Амплитуды колебаний помольной камера ЭШМКТ и соответственно мелющих тел в режимах, близких к резонансу, могут достигать значительно больших значений, чем аналогичные параметры ШМВТ. Причём для получения больших амплитуд колебаний в таких режимах работы ЭШМКТ потребуется возмущающая сила значительно меньшей величины. Квазирезонансные режимы работы ЭШМКТ реализуются путём соответствующего подбора частоты возмущающей силы, согласующейся с резонансной частотой колебательной системы [13].

При работе ЭШМКТ в ходе разрушения и изменения крупности рудного материала неизбежно будут изменяться параметры колебательной системы, что повлечёт соответствующее изменение резонансной частоты и уменьшение амплитуд колебаний. Однако, при регулировании частоты подаваемого на обмотку ТЭМ напряжения появляется возможность постоянно поддерживать работу ЭШМКТ в режимах, близких к резонансу, что в свою очередь сопровождается максимальной производительностью.

При реализации работы ЭШМКТ в колебательном режиме по одной из пространственных координат появляется возможность моделирования работы такого устройства на основе решения системы дифференциальных уравнений движения отдельных элементов колебательной системы [14-19]. Математическое моделирование режимов работы ЭШМКТ с приводом от ТЭМ позволит разработать практические рекомендации, относящиеся к конструкции такой ШМ, параметрам ТЭМ и системе его электропитания, характеристикам пружинного подвеса.

\section{Экспериментальные исследования}

Важным аспектом математического моделирования является определение адекватных параметров (коэффициентов) дифференциальных уравнений движения элементов колебательной системы. Одним из основных параметров уравнений движения являются массы отдельных элементов. Для выбора значений масс элементов колебательной системы ЭШМКТ воспользуемся техническими характеристиками одного из аналогов ШМ, имеющего следующие параметры:

- Диаметр барабана, мм 900

- Длина барабана, мм 1800

- Рабочий объём барабана, м ${ }^{3} \quad 1,14$

- Масса мельницы, кг 5700

- Мощность электродвигателя, кВт 22,0

Для указанной ШМ по принятым методикам проектирования определены следующие расчётные параметры:

- Диаметр шаров, мм

60

- Количество шаров, шт.

1730

- Объём, занимаемый шарами, м $^{3} \quad 0,26$

- Объём, занимаемый рудным

материалом, $\mathrm{M}^{3} \quad 0,26$

- Macca одного шара, кг $\quad 0,848$

- Масса всех шаров, кг 1467

- Количество слоёв из шаров, шт. 4

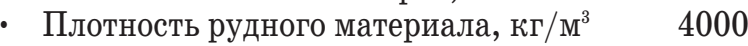

- Масса рудного материала, кг 1040

- Коэффициент использования рабочего объёма помольной камеры, от. ед. $\quad 0,45$

Используя приведённые выше параметры для выбранного аналога, принимаем следующие показатели для экспериментального расчётного варианта ЭШМКТ:

- Диаметр шаров, мм

60

Диаметр помольной камеры, мм 900

- Количество шаров, шт. 1730

Из соображений оптимальности организации процесса колебаний принимаем вертикальное исполнение ЭШМКТ. Учитывая приведённые показатели экспериментального варианта мельницы и особенности такого исполнения ЭШМКТ при циклической выгрузке готового продукта через дно можно определить другие характеристики конструкции экспериментального образца ЭШМКТ, которые потребуются для определения коэффициентов дифференциальных уравнений движения:

- Высота помольной камеры, м, не менее 1,02

- Количество слоёв мелющих тел, шт. 10

- Рабочий объём камеры, $\mathbf{m}^{3} \quad 0,66$

- Масса мельницы, кг, не более (эскизная) 2500

- Количество мелющих тел в одном слое, шт. 173

Комплекс определённых таким образом показателей ЭШМКТ позволяет разработать общее кон- 
структивное решение ШМ, которое приведено на рис. 1. Распределение шаров и рудного материала по объёму помольной камеры на рис. 1 показано условно.

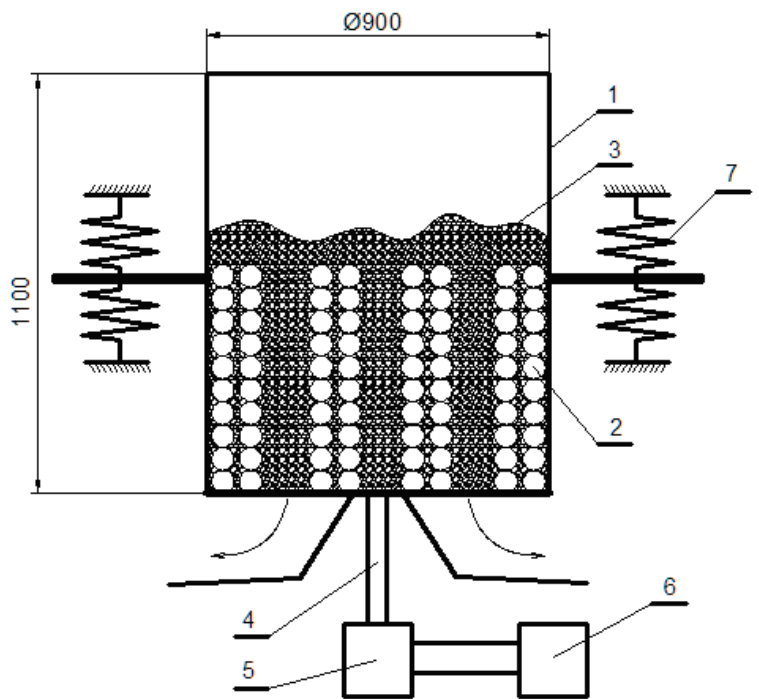

Puc. 1. Общая конструктивная схема ЭШМКТ: 1 - корпус помольной камеры; 2 - мелющие тела (шары); 3 - рудный материал; 4-шток; 5-тЭМ;6-блок питания; 7 - пружинный подвес

Fig. 1. General construction diagram of an electro-magnetic ball mill of a vibrating type: 1 is the grinding chamber frame: 2 are the grinding bodies (balls); 3 is the ore; 4 is the shaft; 5 is the tractive electromagnet; 6 is the power unit; 7 is the spring suspension

Как следует из рис. 1 , корпус помольной камеры - 1 ЭШМКТ с заполненными шарами - 2 и кусками - 3 конгломерата рудного материала совершает колебания в вертикальной плоскости под действием возмущающей силы, передаваемой на корпус через шток - 4 от ТЭМ - 5. От блока питания - 6 на ТЭМ - 5 подаётся переменное напряжение, которое регулируется по величине и частоте. Для пружинного подвеса помольной камеры ЭШМКТ могут быть использованы цилиндрические пружины сжатия -7 .

Важнейшим позитивным показателем разработанной общей схемы ЭШМКТ является повышенное значение коэффициента использования рабочего объёма, который может быть равен от 0,7 до 0,8 .

Колебательная система с общей массой, равной сумме масс помольной камеры, всех шаров и общей массе рудного материала, имеет собственную частоту, равную

$$
\omega_{0}=\sqrt{\frac{C}{M}},
$$

где $C$ - суммарная жёсткость пружинного подвеса; $M$ - общая масса всех совершающих колебания элементов.

Можно предположить, что при определённых параметрах колебаний помольной камеры ЭШМКТ будет происходить отрыв шаров нижнего слоя от дна помольной камеры. Также возможно образование разрывов между слоями шаров и кусками рудного материала. Такое движение отдельных элементов колебательной системы будет сопровождаться ударами кусков рудного материала с мелющими телами друг с другом и с элементами конструкции помольной камеры. Многочисленно повторяющиеся удары будут приводить к разрушению кусков рудного материала [20]. Регулированием величины и частоты напряжения, подаваемого на ТЭМ, можно поддерживать режим работы ЭШМКТ, близкий к резонансному, с амплитудой колебаний корпуса помольной камеры, обеспечивающей получение требуемой крупности рудного материала с высокой производительностью при меньших энергозатратах.

Объективное моделирование работы сложной механической системы с очень большим количеством не связанных друг с другом отдельных элементов, что и представляет собой конструкция ЭШМКТ, является практически неразрешимой задачей. Однако на основе некоторых корректных допущений, относящихся к механике движения элементов ШМ, при математическом моделировании можно получить отдельные и важные практические рекомендации по разработке ЭШМКТ и режимам её работы.

В общем объёме помольной камеры ЭШМКТ можно выделить вертикальный столбец мелющих тел и рудного материала, ограниченный цилиндрическим пространством и представленный на рис. 2.

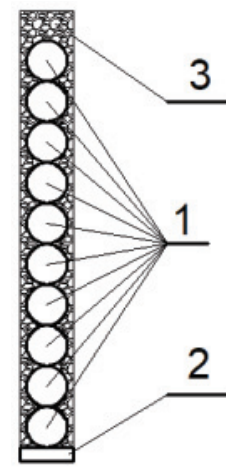

Pис.2. Цилиндрический элемент ЭШМКТ: 1 - мелющие тела (цары) в количестве 10 шт.; 2 - часть конструкиии помольной калеры, соответствующая проекции шара на днище; 3 - рудный материал, прилегающий к мелющим телам

Fig. 2. Tubular element of an electro-magnetic ball mill of a vibrating type: 1 is the grinding bodies (balls) (10 items); 2 is the section of a grinding chamber frame, conforming with the ball projection to the bottom; 3 is the ore adjacent to grinding bodies

При математическом моделировании движения составных частей механической системы, представленной на рис. 2, заменим массу десяти отдельных шаров одной массой, равной сумме масс всех этих шаров. Массу рудного материала, связанную с шарами, можно определить из условия 
равенства объёмов всех мелющих тел ШМ и загружаемого рудного материала. Тогда общая масса одного из элементов колебательной системы будет складываться из массы десяти шаров и массы рудного материала, соответствующей указанному числу шаров. Изложенные выше преобразования позволяют разработать расчётную модель колебательной системы, представленной на рис. 3.

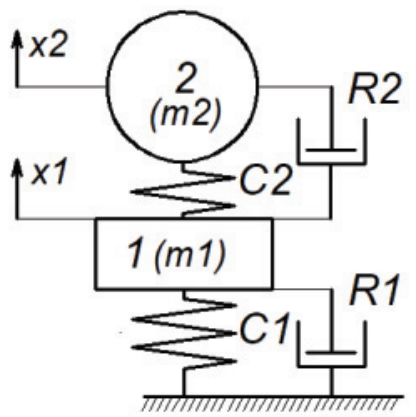

Рис.3. Расчётная схема двухмассовой колебательной системы ЭШMКТ

Fig. 3. Design model of a dual-mass vibration system of an electromagnetic ball mill of a vibrating type

На рис. 3 приняты следующие обозначения: $m 2$ - общая масса всех элементов, образующих тело 2 внутри помольной камеры (шары с рудным материалом); $m 1$ - масса тела 1 , соответствующая определённой части общей массы помольной камеры ЭШМКТ; $C 1$ - жёсткость взаимодействия тела 1 с основанием установки ЭШМКТ; C2 - жёсткость взаимодействия между телами 1 и $2 ; R 1$ - коэффициент вязких потерь в пружинном подвесе ЭШМКТ; $R 2$ - коэффициент вязких потерь при колебаниях тела 2 относительно тела 1.

Согласно описанию рис. 2 ,

$$
m 2=m_{\mathrm{II}}+m_{\mathrm{pM}}=12,21 \kappa \Gamma,
$$

где $m_{\mathrm{m}}=8,82$ кг, масса 10 шаров; $m_{\mathrm{pм}}=3,39$ кг, масса рудного материала.

Массу части общей массы помольной камеры $m 1$ определим из условия обеспечения прочности днища при её толщине не менее 30 мм. Для условий, изложенных в комментариях к рис. 2, масса $m 1$ может составлять расчётную величину не менее 4,0 кг.

Жёсткость взаимодействия между днищем помольной камеры и мелющими телами $C 2$ является переменной величиной, изменяющейся скачком. При колебаниях тел 1 и 2 и отсутствии зазора между ними, что практически соответствует условиям удара этих тел, и при движении $m 1$ вверх жёсткость $C 2$ можно определить, используя известное значение модуля упругости для стали $E=2 \cdot 10^{11}$ Па. Значение $C 2$ для этого случая и для принятых размеров элементов корпуса рабочей камеры и мелющих тел при математическом моделировании может быть принято равным величине в пределах от $10^{7}$ до $10^{9} \mathrm{H} /$ м. Во всех других случаях движения тел 1 и 2 значение C2 можно принять равным нулю.
Жёсткость $C 1$ при известных массах $m 1$ и $m 2$ для условий резонанса можно определить расчётным путём по выражению (1). Следует обратить внимание на тот факт, что существуют два крайних расчётных значения $C 1$ : жёсткость $C 1_{1}-$ соответствующая колебаниям только тела 1 , и жёсткость $C 1_{1-2}$ - соответствующая совместным колебаниям обоих тел 1 и 2 при их соприкосновении. Для определения расчётного численного значения C1 первоначально примем величину собственной частоты колебательной системы, равную 25 Гц. Значения указанных жёсткостей при такой частоте: $C 1_{1}=103,5 \cdot 10^{3} \mathrm{H} / \mathrm{m} ; C 1_{1-2}=404,2 \cdot 10^{3} \mathrm{H} /$ м. В пересчёте значения общей жёсткости подвеса помольной камеры с рудным материалом эти жёсткости составляют соответственно: $17,25 \cdot 10^{6}$ и $78,87 \cdot 10^{6} \mathrm{H} /$ м. Изготовление цилиндрических пружин с указанными значениями жёсткости является практически не разрешимой задачей. Реально достижимым значением жёсткости пружины при её удовлетворительной долговечности является величина, не превышающая $10^{5} \mathrm{H} /$ м. Учитывая это обстоятельство, следует принять пониженную частоту работы ЭШМКТ уровня не более 15 Гц. В этом случае значение жёсткости $C 1_{1}$, обеспечить которое можно путём параллельной установки нескольких пружин, составит $6,2 \cdot 10^{6} \mathrm{H} /$ м. Принимая во внимание это и то, что важным обстоятельством, влияющим на процессы разрушения рудного материала, являются параметры колебаний мелющих тел и конгломератов руды, в дальнейшем при моделировании будем ориентироваться на значение жёсткости подвеса помольной камеры, соответствующее расчётной схеме рис. 3 , равное $10^{5} \mathrm{H} / \mathrm{м}$.

Математическое моделирование режимов работы ЭШМКТ, представленной на расчётной схеме колебательной системы рис. 3, целесообразно провести для определения возможности и создания необходимых условий, обеспечивающих образование относительных колебаний между массами $m 2$ и $m 1$ с образованием зазоров, приводящих к взаимным ударам.

Колебания тел в схеме рис. 3 совершаются под действием возмущающей силы $F(t)$, создаваемой ТЭМ. Уравнение движения тела 1 с учётом всех сил, действующих на него, выглядит следующим образом:

$$
\begin{gathered}
m 12 \frac{d^{2} x 1}{d t^{2}}+R 1 \frac{d x 1}{d t}-R 2\left(\frac{d x 2}{d t}-\frac{d x 1}{d t}\right)+ \\
+C 1 x 1-C 2(x 2-x 1)+P 1+P 21=F(t)+F 21(t),
\end{gathered}
$$

где $m 12$ - суммарная масса тел 1 и 2 при условии $x 1>x 2 ; x 1$ - смещение тела $1 ; x 2$ - смещение тела 2 ; $P 1$ - сила тяжести тела $1 ; P 21$ - сила тяжести тела 2 , действующая на тело 1 при $x 1>x 2 ; F 21(t)$ - сила инерции тела 2 , действующая на тело 1 при условии $x 1>x 2$ и при движении тела 2 вниз.

Уравнение движения тела 2 с учётом всех сил, действующих на него, выглядит следующим обра30M: 


$$
\begin{gathered}
m 2 \frac{d^{2} x 2}{d t^{2}}+R 2\left(\frac{d x 2}{d t}-\frac{d x 1}{d t}\right)+ \\
+C 2(x 2-x 1)+P 2=F 12(t)+F 2(t),
\end{gathered}
$$

где $P 2$ - сила тяжести тела $2 ; F 12(t)$ - сила инерции тела 1 , действующая на тело 2 при условии $x 1>x 2$ и движения тела 1 вверх; $F 2(t)$ - возмущающая сила $F(t)$, действующая на тело 2 при условии $x 1>x 2$ и движения тела 1 вверх.

После преобразований уравнений (2) и (3) и при указанных выше корректных допущениях система дифференциальных уравнений, позволяющая моделировать режимы работы ЭШМКТ, выглядит следующим образом:

$$
\left\{\begin{array}{l}
\frac{d y 1}{d t}=\frac{\left[\begin{array}{l}
F(t)-P 1-P 21+F 21(t)-R 1 y 1- \\
-C 1 x 1+R 2(y 2-y 1)+C 2(x 2-x 1)
\end{array}\right]}{m 12(t)} ; \\
\frac{d y 2}{d t}=\frac{\left[\begin{array}{l}
F 2(t)+F 12(t)-P 2- \\
-R 2(y 2-y 1)-C 2(x 2-x 1)
\end{array}\right]}{m 2} .
\end{array}\right.
$$

Согласно (4), y1 и $у 2$ соответствуют скоростям движения тел 1 и 2 , а их производные - соответственно ускорениям этих тел. Масса $m 12(t)$ является переменной величиной, изменение которой можно учесть следующим образом: при $x 1>x 2$ $m 12(t)=m 1+m 2$, в противном случае $m 12(t)=m 1$.

Особое внимание при решении системы дифференциальных уравнений (4) следует обратить на соблюдение условий взаимодействия двух практически механически не связанных колеблющихся тел 1 и 2.

Знакопеременную возмущающую силу $F(t)$, создаваемую ТЭМ, при моделировании задаём в соответствие с выражением

$$
F(t)= \pm F m \cos \left(2 \pi f t+\frac{\pi}{2}\right)^{2},
$$

где $F m$ - амплитуда возмущающей силы; $f$ - частота питающего напряжения ТЭМ.

Частота возмущающей силы, действующей на помольную камеру ЭШМКТ и определяемой по выражению (5), в этом случае будет соответствовать двойному значению $f$. Знаки «+» или «-» определяют начальное направление действия возмущающей силы ТЭМ.

Не меньший интерес при анализе режимов работы ЭШМКТ представляют процессы, сопровождающиеся относительными колебаниями отдельных слоёв мелющих тел. Данные процессы можно рассмотреть на основе трёхмассовой колебательной системы, приведённой на рис. 4.

На данной схеме в отличие от рис. 3 представлены два слоя мелющих тел 2 и 3 , взаимодействие которых характеризуется дополнительными параметрами: смещение - $x 3$; жёсткость - $C 3$; коэффициент вязких потерь - $R 3$. Значения жёсткости C3 принимаем аналогичными $C 2$. Коэффициент вязких потерь $R 3$ определяется потерями на трение между телами 3 и 1.
Используя аналогичные предыдущему случаю подходы к анализу взаимодействия элементов колебательной системы рис. 4 , получим систему дифференциальных уравнений (6), решая которую можно анализировать более сложные режимы работы ЭШМКТ. Главной особенностью системы уравнений (6) является наличие возможности проведения анализа процессов, связанных с образованием зазоров между мелющими телами 2 и 3.

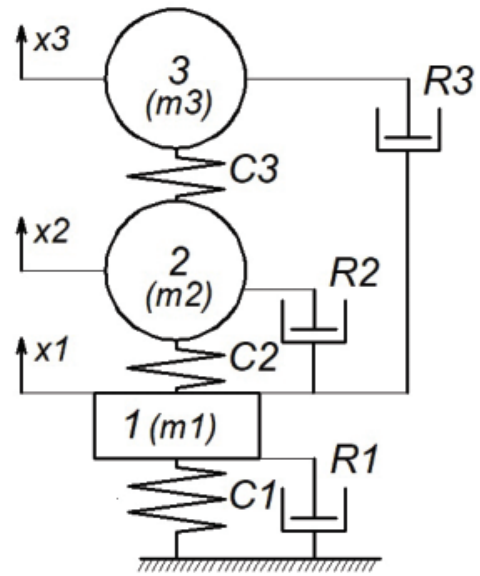

Рис. 4. Расчётная схема трёхмассовой колебательной системы ЭШМКТ

Fig. 4. Design model of a three-mass vibration system of an electromagnetic ball mill of a vibrating type

$$
\left\{\begin{array}{l}
\frac{d y 1}{d t}=\frac{\left[\begin{array}{l}
F(t)-P 1-P 21-P 31+F 21(t)+ \\
+F 31(t)-R 1 y 1-C 1 x 1+ \\
+R 2(y 2-y 1)+C 2(x 2-x 1)
\end{array}\right]}{m 123(t)} ; \\
\frac{d y 2}{d t}=\frac{\left[\begin{array}{l}
F 2(t)-P 2-P 32+F 12(t)+ \\
+F 32(t)-R 2(y 2-y 1)-C 2(x 2-x 1)- \\
-C 3(x 2-x 3)-R 3(y 2-y 3)
\end{array}\right]}{m 23(t)} ; \\
\frac{d y 3}{d t}=\frac{\left[\begin{array}{l}
F 3(t)-P 3+F 13(t)+F 23(t)- \\
-R 3(y 3-y 2)-C 2(x 3-x 2)
\end{array}\right]}{m 3} .
\end{array}\right.
$$

Система (6) содержит дополнительные переменные: $y 3$ - скорость движения тела $3 ;$ P31 - сила тяжести тела 3 , действующая на тело 1 при $x 1>x 2>x 3$; $F 31(t)$ - сила инерции тела 3 , действующая на тело 1 при $x 1>x 2>x 3$ и $y 2<y 3<0 ; m 3$ - масса тела 3 ; $m 123(t)$ - масса тела 1 с присоединёнными в определённых моментах массами тел 2 и $3 ; m 23(t)$ - масса тела 2 с присоединённой в определённых моментах массой тела 3; P32 - сила тяжести тела 3, действующая на тело 2 при $x 2>x 3 ; F 32(t)$ - сила инерции тела 3 , действующая на тело 2 при $x 2>x 3$ и $y 3<0$; $F 3(t)$ - возмущающая сила, действующая на тело 3 при условии $x 1>x 2>x 3$ и $y 1>y 2>0 ; F 13(t)-$ сила инерции тела 1 , действующая на тело 3 при $x 1>x 2>x 3$ и $y 1>y 2>0 ; F 23(t)$ - сила инерции тела 2 , действующая на тело 3 при $x 2>x 3$ и $y 2>0$. 
Системы дифференциальных уравнений (4) и (6) решаются численным методом Рунге-Кутты. Входными изменяющимися параметрами, влияющими на решения, являются: амплитуда возмущающей силы, значение частоты возмущающей силы, масса помольной камеры ЭШМКТ и суммарная масса мелющих тел и рудного материала. В результате решений систем уравнений (4) и (6) могут быть получены информативные данные об изменениях во времени смещений, скоростей и ускорений тел 1,2 и 3 . Действующее значение мощности, требуемой от ТЭМ, в различных режимах работы ЭШМКТ при математическом моделировании рассчитывается по следующей формуле

$$
P_{\text {дейст }}=\sqrt{\frac{\sum_{i=\frac{(N-n) T_{\text {пер }}}{h}}^{\frac{T_{\text {расч }}}{h}} P_{i}^{2} h}{n T_{\text {пер }}}},
$$

где $P_{i}$ - мгновенное значение мощности, затрачиваемой на колебания тела $1 ; N-$ количество точек расчёта; $n$ - количество точек расчёта для установившегося режима; $T_{\text {расч}}-$ время расчёта; $T_{\text {пер }}-$ период колебаний; $h$ - шаг расчёта.

\section{Результаты}

Для моделирования режимов работы ЭШМкТ по уравнениям (4) необходимо определиться с коэффициентами вязких потерь $R 1, R 2$. Потери в пружинном подвесе, определяющие величину $R 1$, при небольших амплитудах колебаний тела 1 составляют достаточно небольшую величину. Имеющаяся информация о работе реальных пружинных подвесов с массами, аналогичными массам тел 1 и 2 , позволяет принять значение $R 1$, не превышающее величину $10 \mathrm{\kappa г} / \mathrm{c}$. Потери на вязкое трение при колебаниях тела 2 будут, естественно, больше, чем $R 1$. Данные потери возникают при точечных соприкосновениях мелющих тел, представленных на рис. 2, с рядом расположенными мелющими телами, кусками рудного материала и элементами конструкции помольной камеры. Реально возможные значения сопротивления $R 2$, предположительно, могут составлять не менее $200 \mathrm{\kappa г} / \mathrm{c}$.

Таким образом, нами определены основные данные, необходимые для моделирования различных режимов работы ЭШМКТ по уравнениям (4) и (6).

Первоначально решим задачу определения условий, при которых могут возникать значимые зазоры между телами 1 и 2 в системе рис. 3 . Графики изменения $x 1$ и $x 2$ во времени при $F m=150 \mathrm{H}$, $m 1=4,21 \mathrm{\kappa r}, \quad m 2=12,21 \mathrm{\kappa \Gamma}, \quad C 1=100 \cdot 10^{3} \mathrm{H} / \mathrm{M}$, $C 2=10^{8} \mathrm{H} / \mathrm{m}, R 1=10 \mathrm{\kappa} / \mathrm{c}, R 2=400 \mathrm{\kappa} \Gamma / \mathrm{c}, f_{\text {рез }}=16,4$ Гц приведены на рис. 5. Частота колебаний 16,4 Гц была определена расчётами и соответствует резонансу, что иллюстрируется рис. 6.

Как видно из рис. 5 для приведённых выше данных при работе ЭШМКТ в режиме резонанса между днищем помольной камеры и мелющими телами возникают существенные зазоры (до 5 мм), чередующиеся со столкновениями этих тел, при- чём столкновения происходят на каждом периоде колебаний помольной камеры. Амплитудно-частотные характеристики рис. 6 свидетельствуют 0 наличии резонанса на частоте 16,4 Гц.

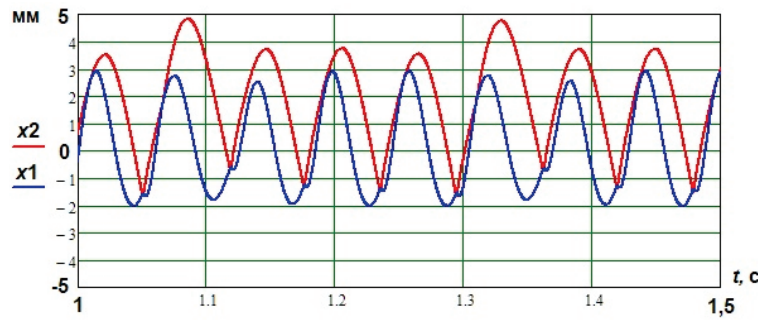

Pис. 5. Смещение тел 1 и 2 при частоте $f=16,4$ Ги

Fig. 5. 1 and 2 bodies shift on a frequency of $f=16,4 \mathrm{~Hz}$

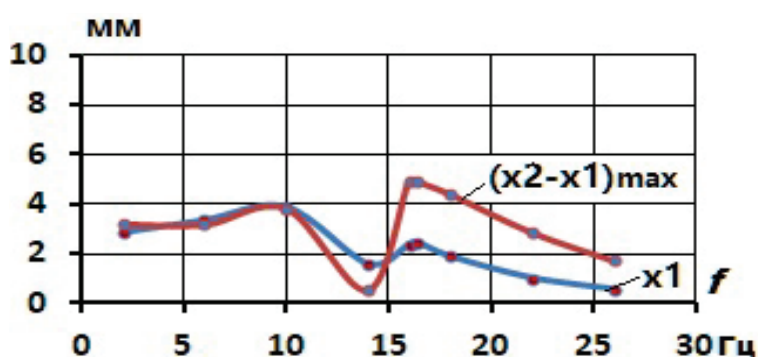

Puc.6. Алплитудно-частотные характеристики ЭШМКТ (puc. 3)

Fig. 6. Amplitude-frequency response of an electro-magnetic ball mill of a vibrating type (Fig. 3)

Для сравнения на рис. 7 приведены аналогичные зависимости при частоте возмущающей силы 40 Гц. На данной частоте возмущающей силы амплитуда колебаний тела 2 резко уменьшается, что приводит к значительному уменьшению максимального зазора между телами 1 и 2 , который не превышает 1,0 мм.

На рис. 8 приведено изменение величины данного зазора, соответствующее разности в мгновенных значениях $x 2$ и $x 1$. Максимальное расчётное значение образующегося зазора межу нижним слоем шаров и днищем помольной камеры при работе ЭШМКТ с указанными выше параметрами составляет порядка 5,0 мм при необходимой мощности, рассчитываемой по формуле (7), равной 2,18 кВт.

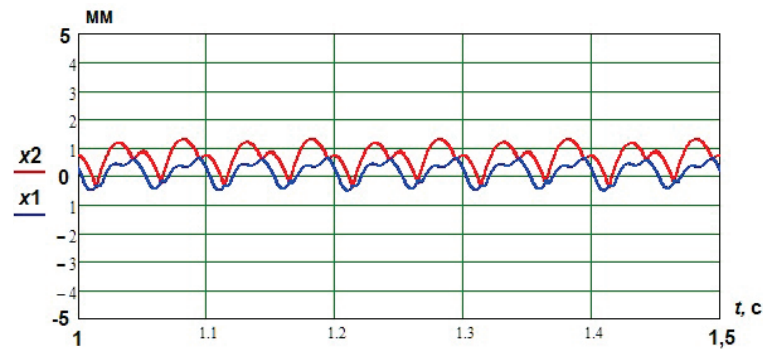

Рис. 7. Смещение тел 1 и 2 при $f=40$ Ги

Fig. 7. 1 and 2 bodies shift with $f=40 \mathrm{~Hz}$

Далее расчёты показывают, что изменяя величину амплитуды возмущающей силы $F m$, можно менять режимы работы ЭШМКТ, тем самым прог- 
нозировать условия, при которых возникает и устанавливается определённый зазор между телами 1 и 2. На рис. 9 приведен график изменения $\Delta x_{\max }=x 2(t)-x 1(t)$ при варьировании амплитуды возмущающей силы $F m$ на частоте 16,4 Гц.

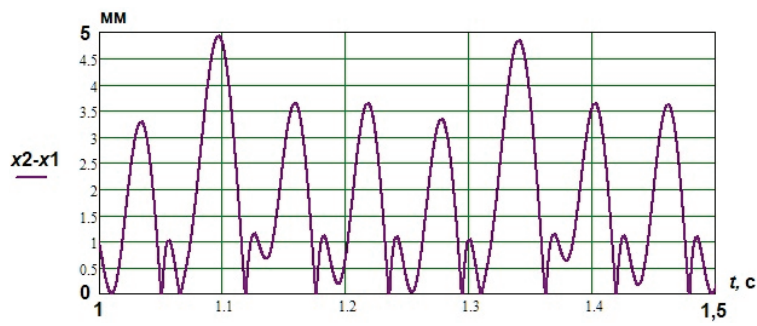

Pис. 8. Изленение зазора между телами 2 и 1

Fig. 8. Clearance modification between bodies 2 and 1

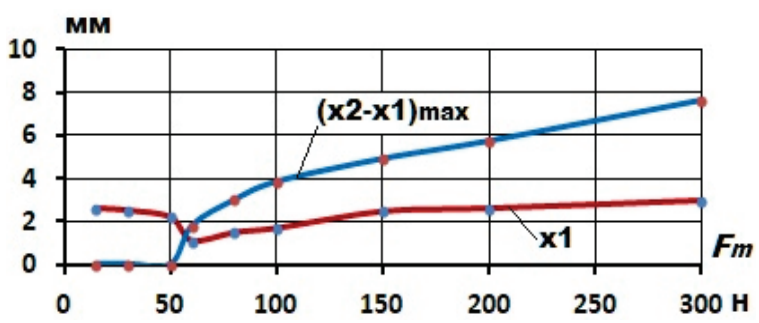

Puс. 9. Зависимость $\Delta x_{\max }$ om Fm

Fig. 9. Dependence of $\Delta x_{\max }$ on Fm

Как следует из данного рисунка, при $F m<50 \mathrm{H}$ тела 1 и 2 совершают колебания практически без образования зазоров меду ними. Далее при увеличении $F m$ образующийся зазор $\Delta x_{\max }$ возрастает практически по линейному закону. Следовательно, режимы работы ЭШМКТ при постоянной частоте можно регулировать изменение $F m$, добиваясь определённых значений зазора между телами 1 и 2 , от величины которого зависит производительность мельницы. При $F m>150 \mathrm{H}$ величина $\Delta x_{\max }$ становиться достаточно большой, что позволяет принять данное значение $\mathrm{Fm}$ для рассматриваемого варианта ЭШМКТ как основное рабочее, для которого максимальный зазор между днищем помольной камеры и нижним слоем мелющих тел составляет не менее 5,0 мм при подводимой мощности, определяемой по выражению (7) и равной $2,18 \mathrm{\kappa B \textrm {T }}$

На рис. 10 представлены результаты расчётов режимов работы ЭШМКТ при изменении механического сопротивления $R 2$. При незначительных величинах $R 2$ зазор между мелющими телами и днищем помольной камеры ЭШМКТ может возрастать до нескольких десятков миллиметров. Надо отметить, что эти данные при отмеченных выше ограничениях носят чисто теоретический характер. Практически величина сопротивления $R 2$ в реальных условиях, вероятнее всего, составляет не менее 200 кг/с и выше.

На рис. 11 приведен график изменения величины $\Delta x_{\max }$ от изменения массы помольной камеры, свидетельствующий о некотором увеличении зазоров между мелющими телами и днищем помоль- ной камеры при увеличении массы $m 1$, однако это наблюдается до определённого предела. Для рассмотренного случая масса $m 1$ не должна превышать 7,2 кг, что соответствует общей массе помольной камеры в 1250 кг.

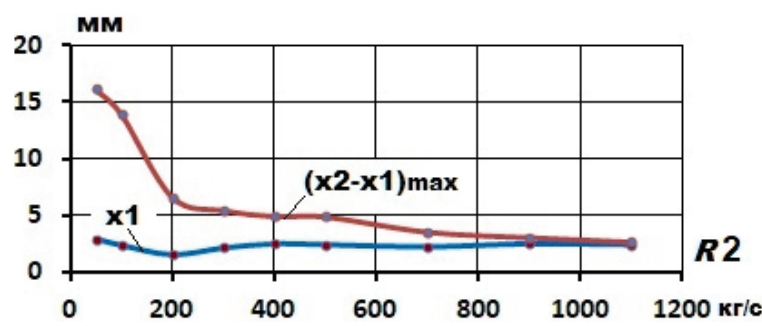

Puс. 10. Зависилость $\Delta x_{\max }$ om R2 при Fm=150 H

Fig. 10. Dependence of $\Delta x_{\max }$ on R2 with Fm=150 N

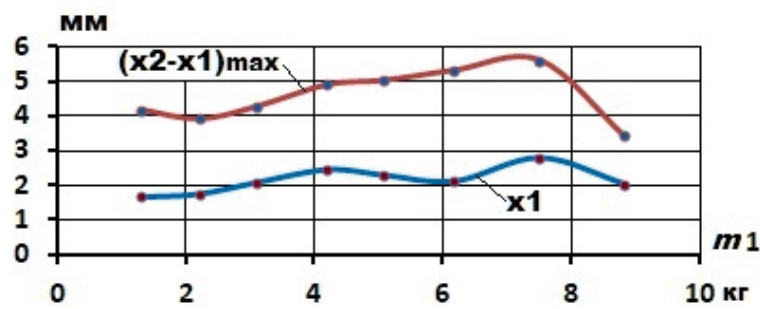

Puc. 11. Зависилость $\Delta x_{\max }$ om $\mathrm{m} 1 \mathrm{npu} \mathrm{Fm}=150 \mathrm{H}$

Fig. 11. Dependence of $\Delta x_{\max }$ on $m 1$ with Fm=150 N

Особый интерес представляет математическое моделирование режимов работы трёхмассовой колебательной системы ЭШМКТ (рис. 4) с позиций определения условий образования зазоров между днищем помольной камеры и нижним слоем мелющих тел, а также между отдельными слоями мелющих тел.

На рис. 12 приведены графики изменения $x 1$, $x 2$ и $x 3$ при следующих параметрах: $F m=150 \mathrm{H}$, $m 1=4,21$ кг, $m 2=1,21$ кг, $m 3=11,0$ кг, $C 1=100 \cdot 10^{3} \mathrm{H} / \mathrm{M}$, $R 1=10 \mathrm{\kappa \Gamma} / \mathrm{c}, \quad R 2=40 \mathrm{\kappa \Gamma} / \mathrm{c}, \quad C 2=C 3=10^{8} \mathrm{H} / \mathrm{m}$, $R 3=360$ кг $/ \mathrm{c}, f_{\text {рез }}=20,4$ Гц. При этом тело 2 образует один нижний слой мелющих тел с соответствующим количеством рудного материала, а тело 3 все остальные девять слоёв мелющих тел и соответствующий им рудный материал. Частота $f=20,4$ Гц соответствует квазирезонансному режиму работы ЭШМКТ по рис. 4.

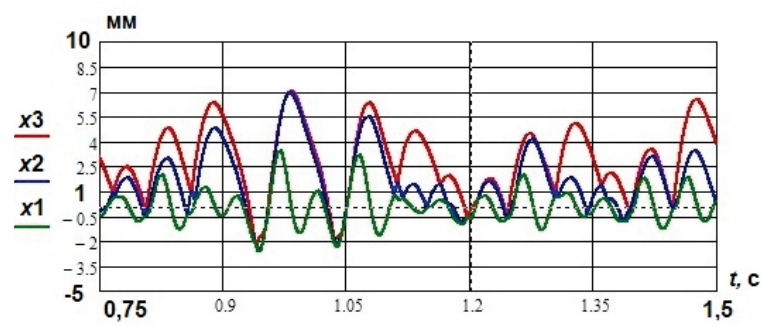

Pис. 12. Смещение $x 1, x 2, x 3$ при $f=20,4$ Ги

Fig. 12. $x 1, x 2, x 3$ shift with $f=20,4 \mathrm{~Hz}$

На рис. 13, 14 приведены соответствующие изменения зазоров между телами 1,2 и 3. Как видно из представленных рисунков, величины зазоров между 
телами 1, 2 и 3 для рассматриваемой трёхмассовой системы превышают зазоры, образующиеся в двухмассовой системе. При этом наблюдается некоторая нестабильность циклов для обоих случаев.

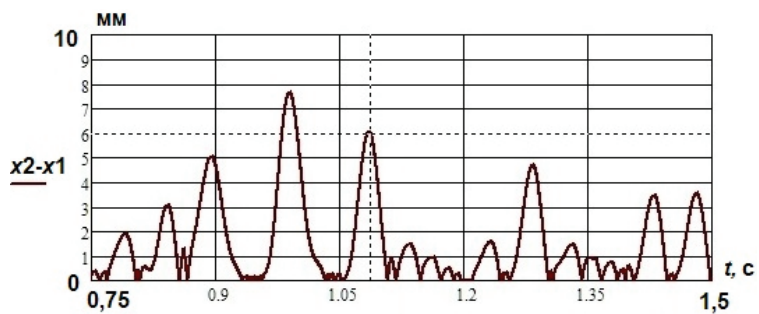

Pис. 13. Изленение зазора между телами 2 и 1

Fig. 13. Clearance modification between bodies 2 and 1

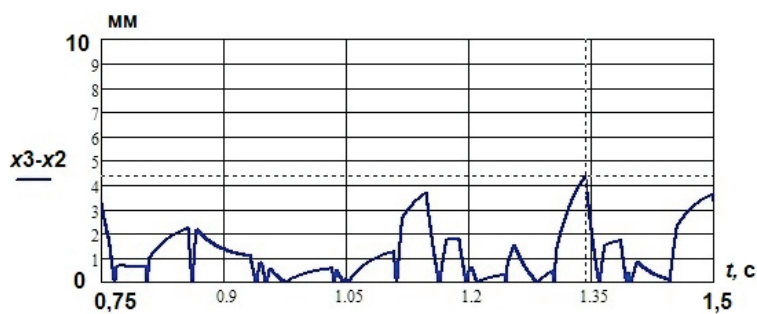

Рис. 14. Изленение зазора между телами 3 и 2

Fig. 14. Clearance modification between bodies 3 and 2

Проведённые расчёты при изменении частоты питающего напряжения выявили ещё одну особенность трёхмассовой системы. В данной системе по отношению к двухмассовой системе резонанс смещается в сторону большей частоты, равной 20,4 Гц, что иллюстрируется рис. 15.

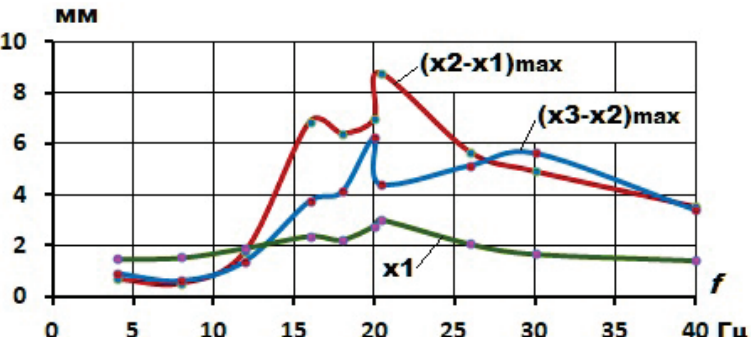

Pис. 15. Алплитудно-частотные характеристики ЭШМКТ (puc. 4)

Fig. 15. Amplitude-frequency response of an electro-magnetic ball mill of a vibrating type (Fig. 4 )

Как следует из представленных графиков на рис. $5,7,11$, в определённые моменты происходит соударение между мелющими телами друг с другом и с днищем помольной камеры. На рис. 14,15 приведены графики изменения взаимных скоростей $y 1, y 2$ и $y 3$. В квазирезонансных режимах максимальная скорость соударений днища помольной камеры и нижнего слоя шаров составляет порядка 1,2 м/с, а скорость соударений между слоями шаров - не менее $1,8 \mathrm{~m} / \mathrm{c}$.

Указанные значения скоростей соударений в ЭШМКТ превышают значения относительных скоростей, имеющих место в барабанных ШМ: скорость подъёма шаров по ободу барабана ШМ не превышает 1,0 м/с, скорость падения шаров не превышает 1,5 м/с. Однако конструкция традиционной барабанной ШМ не предполагает встречного движения мелющих тел с приведёнными скоростями и к тому же количество шаров, обладающих такими скоростями существенно ограничено и не превышает 10-15 \% [21]. Для рассматриваемой конструкции ЭШМКТ приведённые значения скорости соударений характерны для всех мелющих тел по всему объёму, что свидетельствует 0 её более высокой эффективности.

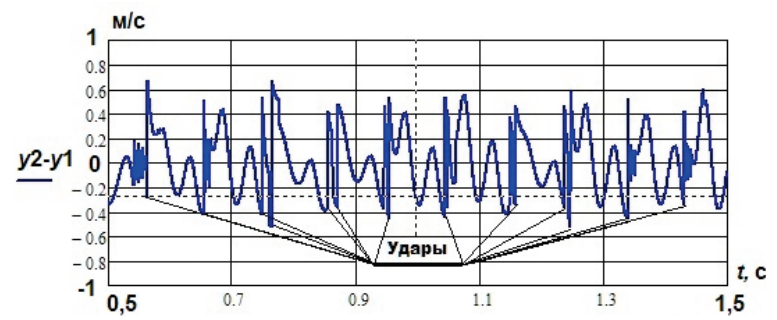

Рис. 16. Относительная скорость тел 2 и 1

Fig. 16. Relative velocity of bodies 2 and 1

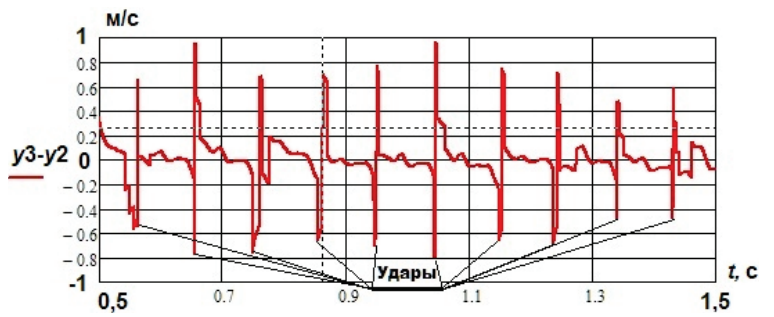

Рис. 17. Относительная скорость тел 3 и 2

Fig. 17. Relative velocity of bodies 3 and 2

Как было отмечено ранее при амплитуде возмущающей силы Fm не менее $150 \mathrm{H}$ формирующиеся в процессе работы ЭШМКТ зазоры между телами 1,2 и 3 являются достаточными для эффективной работы. Мощность ТЭМ, создающего требуемую возмущающую силу, при этом составляет не более 4,0 кВт, что в пять раза меньше, чем для выбранного ранее аналога ШМ.

Следовательно, принимая во внимание приведённые результаты математического моделирования, вполне логичным и достоверным является предположение о более высокой эффективности рассмотренной конструкции ЭШМКТ по сравнению с барабанными ШМ.

\section{Выводы}

1. Разработанная математическая модель позволяет осуществлять анализ работы ЭШМКТ в широком диапазоне изменения амплитуды и частоты возмущающей силы с целью определения условий создания квазирезонансного режима при различных параметрах колебательной системы. Квазирезонансный режим работы ЭШМКТ характеризуется высокими скоростями соударений мелющих тел друг с другом и с элементами помольной камеры, что предопределяет более высокую эффективность процес- 
сов измельчения рудного материала. Регулирование амплитуды и частоты возмущающей силы позволяет оптимизировать процесс получения конечного продукта требуемой крупности с наименьшими затратами энергии.

2. Коэффициент использования рабочего объёма помольной камеры ЭШМКТ практически в два раза превышает аналогичное значение для барабанной ШМ при значительном сокращении затрат электроэнергии. Прогнозируемый расход электроэнергии ЭШМКТ составляет не более 15,0 кВт на тонну готовой продукции. Зна-

\section{СПИСОК ЛИТЕРАТУРЫ}

1. Вопросы оптимизации процесса дробления упорных руд / В.С. Портнов, В.М. Юров, А.К. Турсунбаева, Р.К. Камаров, А.Д. Мехтиев, С.Е. Орынгожина / Фундаментальные исследования. - 2012. - № 9. - С. 167-170.

2. Ханин С.И. Разработка научных основ проектирования шаровых мельниц с энергообменными и классифицирующими устройствами: дис. ... д-ра техн. наук. - Белгород, 2016. $483 \mathrm{c.}$

3. Багдасарян М.К. К определению полезной мощности усовершенствованной конструкции барабанной мельницы // Горное оборудование и электромеханика. - 2010. - № 11. - С. 45-48.

4. Copper ore grinding in a mobile vertical roller mill pilot plant D. Altun, C. Gerold, H. Benzer, O. Altun, N. Aydogan // International Journal of Mineral Processing. - 10 March 2015. V. 136 . - P. 32-36.

5. Effect of different grinding media composition on the flotation of a PGM ore / Z.G. Song, K.C. Corin, J.G. Wiese, C.T. O'Connor // Minerals Engineering. - 1 August 2018. - V. 124. - P. 74-76.

6. Friction and wear of liner and grinding ball in iron ore ball mill / Yu-xing Peng, Xu Ni, Zhen-cai Zhu, Zhang-fa Yu, Jie Xu // Tribology International. - November 2017. - V. 115. - P. 506-517.

7. De Oliveira Alessandro L.R., Tavares L.M. Modeling and simulation of continuous open circuit dry grinding in a pilot-scale ball mill using Austin's and Nomura's models // Powder Technology. - December 2018. - V. 340. - P. 77-87.

8. Quantifying variability of ore breakage by impact - Implications for SAG mill performance / F. Faramarzi, V. Jokovic, R. Morrison, S.S. Kanchibotla // Minerals Engineering. - October 2018. V. 127. - P. 81-89.

9. Fengnian Shi, Weiguo Xie. A specific energy-based ball mill model: From batch grinding to continuous operation // Minerals Engineering. - February 2016. - V. 86. - P. 66-74.

10. Оценка эффективности виброизмельчения минеральных материалов применительно $\mathrm{k}$ твёрдым отходам фтороводородного производства / Ю. М. Федорчук, В.А. Данекер, А.А. Волков, А.М. Адам, Л.А. Аниканова // Международный журнал при- чения тяговых усилий, создаваемых ТЭМ, при этом должны быть не менее 5,0 Н на один килограмм общей загрузки помольной камеры.

3. Рекомендуемое значение частоты возмущающей силы, зависящей от частоты питающего напряжения ТЭМ, определяется соотношением масс незаполненной помольной камеры, массой её технологической загрузки с мелющими телами и жёсткостью пружинного подвеса камеры. По техническим и технологическим характеристикам оптимальная частота работы ЭШМКТ находится в диапазоне от 10 до 20 Гц.

кладных и фундаментальных исследований. - 2016. - № 1. C. $325-330$.

11. Булгаков Е.Б. Вертикальная вибрационная мельница: дис. ... канд. техн. наук. - Белгород, 2008. - 166 с.

12. Operational parameters affecting the vertical roller mill performance / D. Altun, H. Benzer, N. Aydogan, C. Gerold // Minerals Engineering. - April 2017. - V. 103-104. - P. 67-71.

13. Данекер В.А., Рикконен С.В. Приготовление и коррекция показателей бурового раствора технологией и оборудованием BCMA // Известия Томского политехнического университета. Инжиниринг георесурсов. - 2017. - Т. 328. - № 7. - С. 86-92.

14. Быховский И.И. Основы теории вибрационной техники. - М.: Машиностроение, 1969. - 363 с.

15. Ханин С.И. Математическая модель процесса движения мелющих тел в трубной мельнице с винтовыми устройствами // Вестник Белгородского государственного технического университета имени В.Г. Шухова. - 2009. - № 3. - С. 127-130.

16. Мейсснер П., Плохбергер Т., Бем А. Определение параметров помола в вертикальных мельницах // Цемент и его применение. - 2012. - № 6. - С. 104-110.

17. Пелевин А.Е., Мушкетов Ант.Анд. Кинетика измельчения классов крупности титаномагнетитовой руды // Известия вузов. Горный журнал. - 2014. - № 3. - С. 91-96.

18. Quantifying variability of ore breakage by impact - Implications for SAG mill performance / F. Faramarzi, V. Jokovic, R. Morrison, S.S. Kanchibotla // Minerals Engineering. - October 2018. V. 127. - P. 81-89.

19. Влияние структуры системы измельчения шаровой мельницей на ее производительность / С.И. Шувалов, Г.Г. Михеев, А.А. Веренин, Н.С. Асташов // Цемент и его применение. 2011. - № 5. - C. 22-24.

20. Ханин С.И. Характеристики процесса движения мелющих тел в цилиндрическом корпусе шаровой барабанной мельницы // Вестник Белгородского государственного технического университета имени В.Г. Шухова - 2012. - № 3. - С. 91-94.

Поступила 28.02.2019 2.

\section{Информация об авторах}

Данекер В.А., кандидат технических наук, доцент отделения электроэнергетики и электротехники Инженерной школы энергетики Национального исследовательского Томского политехнического университета. 
UDC 621.926.55

MATHEMATICAL SIMULATION OF OPERATIONAL MODES OF A VIBRATING BALL MILL

\author{
Valery A. Daneker, \\ vad@tpu.ru \\ National Research Tomsk Polytechnic University, \\ 30, Lenin avenue, Tomsk, 634050, Russia.
}

The relevance of the research is caused by the need to develop highly-efficient resource-saving equipment, used for kibbling and grinding ore in mining industry. One of the possible solution is the development of ball mills of a vibrating type with AC tractive electromagnet. Connecting a tractive electromagnet to AC voltage source enables a long-range adjustment of operational mode of a ball mill and ensures its operation in quasi-resonant regimes. Mathematical simulation of operational modes of a ball mill of a vibrating type allows determining standards for a vibratory system when changing frequency and value of power supply and generating guidelines to be considered in production prototypes design. Additionally, operation of a ball mill of a vibrating type in quasi-resonant regimes is followed by significant reduction of energy consumption along with productivity gain.

The aim of the research is to develop a mathematical model of operation of a ball mill of a vibrating type with a tractive electromagnet powered by a variable frequency power supply; in terms of the developed mathematical model to calculate the operational modes of a ball mill of a vibrating type and generate the guidelines for use in developing a ball mill.

Methods. Mathematical simulation of operational modes of a ball mill was carried out by a numerical solution of a differential system of elements movement in a vibratory system. Vibratory system parameters were calculated with regard to objective technical data of the similar equipment produced.

Results. The mathematical model of a ball mill of a vibrating type allows determining a range of effective tractive efforts and power supply frequencies of a tractive electromagnet, relevant to a quasi-resonant operation mode when changing vibratory system parameters. With the appropriate quasi-resonant frequency span and masses of elements of a vibratory system we can determine tractive electromagnet parameters, design and specification of a spring suspension of a ball mill.

Key words:

Ore, kibbling, grinding, ball mill, vibratory mill, vibratory system, tractive electromagnet, resonance.

\section{REFERENCES}

1. Portnov V.S., Yurov V.M., Tursunbaeva A.K., Kamarov R.K., Mekhtiev A.D., Oryngozhina S.E. Optimization of refractory ores breaking. Fundamental studies, 2012, no. 9, pp. 167-170. In Rus.

2. Khanin S.I. Razrabotka nauchnykh osnov proektirovaniya sharo vykh melnits s energoobmennymi i klassifitsiruyushchimi ustroystvami. Dis. Kand. nauk [Development of scientific basics for designing ball mills with energy-exchanged devices and circuit guards. Cand. Diss.]. Belgorod, 2016. 483 p.

3. Bagdasaryan M.K. On determining useful power of a drum mill of an improved design. Mining equipment and electromechanics, 2010, no.11, pp. 45-48. In Rus.

4. Altun D., Gerold C., Benzer H., Altun 0., Aydogan N. Copper ore grinding in a mobile vertical roller mill pilot plant. International Journal of Mineral Processing, 10 March 2015, vol. 136, pp. $32-36$.

5. Song Z.G., Corin K.C., Wiese J.G., O'Connor C.T. Effect of different grinding media composition on the flotation of a PGM ore. Minerals Engineering, 1 August 2018, vol. 124, pp. 74-76.

6. Yu-xing Peng, Xu Ni, Zhen-cai Zhu, Zhang-fa Yu, Jie Xu. Friction and wear of liner and grinding ball in iron ore ball mill. Tribology International, November 2017, vol. 115, pp. 506-517.

7. De Oliveira Alessandro L.R., Tavares L.M. Modeling and simulation of continuous open circuit dry grinding in a pilot-scale ball mill using Austin's and Nomura's models. Powder Technology, December 2018, vol. 340, pp. 77-87.

8. Faramarzi F., Jokovic V., Morrison R., Kanchibotla S.S. Quantifying variability of ore breakage by impact - Implications for SAG mill performance. Minerals Engineering, October 2018, vol. 127, pp. 81-89.

9. Fengnian Shi, Weiguo Xie. A specific energy-based ball mill model: from batch grinding to continuous operation. Minerals Engineering, February 2016, vol. 86, pp. 66-74.
10. Fedorchuk Yu.M. Evaluating the effectiveness of vibroizmelcheniya mineral materials applied to solid waste production of hydrogen fluoride. Mezhdunarodny zhurnal prikladnykh i fundamentalnykh issledovaniy, 2016, no. 1, P. 3, pp. 325-330. In Rus.

11. Bulgakov E.B. Vertikalnaya vibratsionnaya melnitsa. Dis. Kand. nauk [A vertical jar mill. Cand. Diss.]. Belgorod, 2008. 166 p.

12. Altun D., Benzer H., Aydogan N., Gerold C. Operational parameters affecting the vertical roller mill performance. Minerals Engineering, April 2017, vol. 103-104, pp. 67-71.

13. Daneker V.A., Rikkonen S.V. Preparation and correction of drilling mud by technology and equipment of vibro-jet-magnetic activation. Bulletin of the Tomsk Polytechnic University. Geo Assets Engineering, 2017, vol. 328, no. 7 pp. 86-92. In Rus.

14. Bykhovskiy I.I. Osnovy teorii vibratsionnoy tekhniki [Fundamentals of vibration engineering]. Moscow, Mashinostroenie Publ., 1969. $363 \mathrm{p}$.

15. Khanin S.I. Mathematical model of driving grinding bodies in tubular mills with screwdowns. Bulletin of Belgorod state engineering university named after V.G. Shukhov, 2009, no. 3, pp. 127-130. In Rus.

16. Meissner P., Plokhberger T., Bem A. Opredelenie parametrov pomola $\mathrm{v}$ vertikalnykh melnitsakh [Determining grinding properties in vertical jar mills]. Concrete and its use, 2012, no. 6, pp. 104-110.

17. Pelevin A.E., Mushketov A.A. Grinding kinetics of grain-size classes of titanium magnetite ore. News of Higher Educational Institutions. Journal of mining, 2014, no. 3, pp. 91-96. In Rus.

18. Faramarzi F., Jokovic V., Morrison R., Kanchibotla S.S. Quantifying variability of ore breakage by impact - Implications for SAG mill performance. Minerals Engineering, October 2018, vol. 127 , pp. 81-89.

19. Shuvalov S.I., Mikheev G.G., Verenin A.A., Astashov N.S. Vliyanie struktury sistemy izmelcheniya sharovoy melnitsy na ee proizvoditelnost [Influence of a grinding system structure with a ball 
mill on its productivity]. Concrete and its use, 2011, no. 5, pp. 22-24.

20. Khanin S.I Kharakteristiki protsessa dvizheniya melyushchikh tel $\mathrm{v}$ tsilindricheskom korpuse sharovoy barabannoy melnitsy [Characteristics of driving grinding bodies in a cylindrical body of a ball tube mill]. Bulletin of Belgorod state engineering university named after V.G. Shukhov, 2012, no. 3, pp. 91-94.

Received: 28 February 2019.

\section{Information about the authors}

Valery A. Daneker, Cand. Sc., associate professor, National Research Tomsk Polytechnic University. 